\title{
Experimental evaluation of cyclic fatigue resistance of four different nickel-titanium instruments after immersion in sodium hypochlorite and/or sterilization
}

\author{
Üreyen Kaya BULEM, Ayse Diljin KECECI, Hilmi Egemen GULDAS
}

Süleyman Demirel University, Faculty of Dentistry, Department of Endodontics, Isparta, Turkey.

Corresponding address: Bulem Üreyen Kaya - Süleyman Demirel Üniversitesi, Diş Hekimliği Fakültesi - Endodonti AD 32260 Kampüs - Isparta - Turkey Phone: +90 2462113705 - Fax: +90 2462370607 - e-mail: bureyen@hotmail.com

Submitted: January 17, 2013 - Modification: May 28, 2013 - Accepted: August 12, 2013

\section{ABSTRACT}

$\mathrm{N}$ iTi instruments have a high risk of separation due to torsional or flexural fatigue (cyclic fatigue). Chemomechanical preparation, cleaning procedures, chemical disinfection and sterilization cause the corrosion of endodontic instruments that may weaken the fracture resistance of the instruments. Objective: To assess the effects of $\mathrm{NaOCl}$ immersion and autoclave sterilization on the cyclic fatigue resistance of ProFile, FlexMaster, Mtwo and TwistedFiles NiTi instruments (tip size 25, 0.06 taper, $n=160$ ). Material and Methods: The instruments ( $n=10$ for each subgroup) were dynamically immersed in $\mathrm{NaOCl}$; immersed in $\mathrm{NaOCl}$ and sterilized in one autoclave cycle; 5 cycles immersed in $\mathrm{NaOCl}$ and sterilized in autoclave and not immersed in $\mathrm{NaOCl}$ and not sterilized (control group). Dynamic cyclic fatigue resistance was tested. The number of cycles to failure (NCF) were statistically analyzed $(P<0.05)$. Results: Kruskall-Wallis test indicated significant differences among the tested instruments in terms of NCF $(P=0.000)$. The mean NCF of Mtwo (556.75) was higher than that of the Twisted File, Flexmaster and ProFile, 483.1, 376.12, 365.25, respectively. $\mathrm{NaOCl}$ immersion and autoclave sterilization have no effect on the NCF values of the tested instruments $(P>.05)$. Conclusions: Cyclic fatigue resistance of the tested NiTi instruments cannot be adversely affected by $\mathrm{NaOCl}$ immersion and autoclave sterilization. Production process (TwistedFiles) or design (Twisted Files, FlexMaster, Mtwo and ProFile) of the instruments can influence their cyclic fatigue resistance.

Key words: Endodontics. Fractures. Sterilization. Stress. Sodium chloride.

\section{INTRODUCTION}

Nickel-titanium (NiTi) instruments are very popular and provide many advantages during root canal treatment. However, NiTi instruments have a high risk of separation due to torsional or flexural fatigue (cyclic fatigue) ${ }^{23}$. Fractures due to cyclic fatigue occur because of metal fatigue and are more prevalent in curved root canals. The instrument rotates freely at the curvature without binding, so that the tensile and compressive stresses are concentrated at the point of maximum flexure. Resistance to fracture prior to an actual fracture is determined by the number of cycles in which an instrument operates under a specific loading condition ${ }^{31}$. When the tip or some part of the file binds in the root canal during rotation, torsional failure occurs ${ }^{23}$.

An important factor that may weaken the fracture resistance is corrosion. Chemomechanical preparation, cleaning procedures, chemical disinfection, and sterilization cause the corrosion of endodontic instruments ${ }^{17}$. Sodium hypochlorite $(\mathrm{NaOCl})$ is the most commonly used irrigant in endodontic treatment ${ }^{2}$, and its active $\mathrm{ClO}$ - ions have the potential to increase corrosion ${ }^{22}$. $\mathrm{NaOCl}$ selectively removes nickel from the instrument surface and causes micropitting 22 . These are the areas where stress gets concentrated, forming cracks $^{22}$ and increasing the probability of fatigue 
failure ${ }^{26}$. Environmental conditions affect crack initiation and propagation processes ${ }^{26}$. The corrosive effect of $\mathrm{NaOCl}$ on endodontic NiTi instruments has been studied previously only in a static mode and for long contact times, which did not simulate actual clinical conditions ${ }^{10}$.

The resistance to cyclic fatigue of $\mathrm{NiTi}$ rotary instruments can be increased via improvements in the manufacturing process or by the use of new alloys with superior mechanical properties. Twisted Files (SybronEndo, Orange, CA, U.S.A.), which were recently introduced, are reported to have better physical and mechanical properties than traditional NiTi instruments. They are produced with a special form of thermomechanical processing, which yields a new, heat-treated NiTi instrument that contains a martensitic phase stable superelastic alloy under clinical conditions ${ }^{7,13}$. It has been reported that the additional heating of $\mathrm{NiTi}$ instruments might increase their flexibility ${ }^{30}$ and microhardness ${ }^{5}$.

There have been many studies on the cyclic fatigue resistance of different NiTi rotary files having different designs or compositions ${ }^{7,8,13}$. There are also several reports related to the effect of autoclave sterilization, for either improving or degrading the surface, mechanical, and cutting properties of various NiTi rotary systems ${ }^{11,24,28,29}$. However, there is no information on the influence of both $\mathrm{NaOCl}$ and autoclave sterilization on the cyclic fatigue resistance of $\mathrm{NiTi}$ rotary instruments. Therefore, the aim of this study was to assess the effect $\mathrm{NaOCl}$ solution and autoclave sterilization has on the cyclic fatigue resistance of three traditional (ProFile, FlexMaster, and Mtwo) NiTi instruments and one heat-treated (Twisted Files) NiTi instrument in conditions similar to those used in clinical practice.

\section{MATERIAL AND METHODS}

Four NiTi rotary instruments - ProFile (Dentsply Maillefer, Ballaigues, Switzerland), FlexMaster (VDW, GmbH, Munich, Germany), Twisted Files (SybronEndo, Orange, CA, U.S.A.), and Mtwo (VDW, $\mathrm{GmbH}$, Munich, Germany) - were selected for the fatigue resistance test; all the instruments were of the same size (\#25, 0.06 taper). Each group included 40 unused instruments that were divided into four subgroups $(n=10)$ to evaluate different protocols.

In group 1 , the instruments were dynamically immersed in $2.5 \% \mathrm{NaOCl}$ for 5 min at room temperature. In group 2, the instruments were immersed in $\mathrm{NaOCl}$, under the same conditions as in group 1, and then subjected to one cycle of autoclave sterilization (Statim 5000S, SciCan, Leutkirch im Allgäu, Germany) at a temperature of $135^{\circ} \mathrm{C}$ for $32.5 \mathrm{~min}(17.5 \mathrm{~min}$ of sterilization and 15 min of drying). In group 3, the instruments were subjected to five cycles of the same test protocols as in group 2. In the control group, the instruments were not immersed in $\mathrm{NaOCl}$ and were not sterilized.

For the dynamic immersion, the endodontic instruments were attached to an endodontic motor and rotated freely at a constant speed (300 rpm for ProFile, FlexMaster, and Mtwo; 500 rpm for Twisted Files) in a small glass container with an amount of the $\mathrm{NaOCl}$ solution that was sufficient to be in contact with the instruments but not the shaft ${ }^{18}$. The files were rinsed with distilled water to neutralize the effect of the $\mathrm{NaOCl}$ and then dried.

A total of 160 instruments, 40 from each subgroup, were tested for dynamic cyclic fatigue resistance. Artificial canals were constructed initially by providing a copper duplicate of each instrument to obtain accurately-sized canals. The copper duplicate

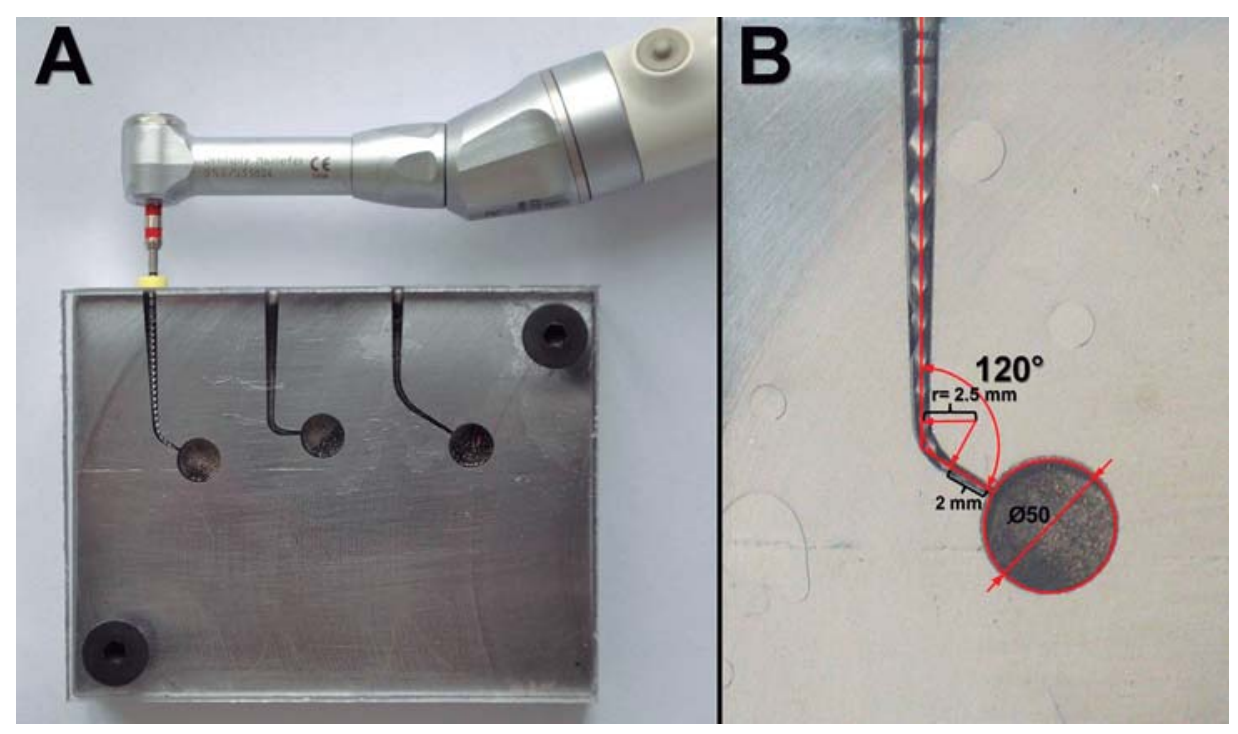

Figure 1-A) Cyclic fatigue testing device and the used canal type B) Trajectory of the size 25/.06 instrument and curvature properties of artificial root canal 
was milled by increasing the original size of the instrument by $0.1 \mathrm{~mm}$, using a computer numeric control machining bench (Ajan CNC, İzmir, Turkey), according to the curvature properties $\left(60^{\circ}\right.$ angle, $2.5 \mathrm{~mm}$ radius, curvature beginning $2 \mathrm{~mm}$ from the tip of the file) that were chosen for this study. It was then constructed in a stainless steel block using the negative mold, using a die-sinking electricaldischarge machining process (Hartford M 65 series, She Hong Industrial Co. Ltd., Taichung, Taiwan). The depth of the artificial canal was machined to the maximum diameter of the instrument plus 0.2 $\mathrm{mm}$, thus allowing the instrument to rotate freely. The artificial canal was also covered with tempered glass to prevent the instruments from slipping out and to allow observation of the instruments. The dental hand-piece was fixed to permit precise placement of each instrument inside the artificial canal and to guarantee the same three-dimensional positions of the instruments (Figures $1 \mathrm{~A}, \mathrm{~B}$ ). All the instruments except the Twisted Files were rotated at a constant speed of $300 \mathrm{rpm}$ using a 16:1 contra angle and powered by a torque-controlled electric motor (X-smart, Dentsply Maillefer, Ballaigues, Switzerland) with back-and-forth axial movements until fracture occurred. The Twisted Files were rotated at a constant speed of $500 \mathrm{rpm}$, as per the manufacturer's recommendation. The amplitude of the axial movements was $3 \mathrm{~mm}$, with about $2 \mathrm{~s}$ for every displacement. High-flow synthetic oil (DME, Gloucester, UK) was poured into the simulated canal after each file change to reduce the friction between the instrument and the metal canal walls.

The time-to-fracture was recorded visually with a 1/100-s chronometer. The number of cycles to failure (NCF) was determined by converting the time required to fracture the instrument into decimal units and then multiplying the time by the number of rotations per minute. The NCF values $(n=40)$ obtained from four subgroups with different protocols were averaged to calculate the mean NCF value for each instrument (ProFile,

Table 1- The mean (SD) number of cycles to failure (NCF)* and fragment lengths in $\mathrm{mm}(\mathrm{SD})$ of tested NiTi instruments

\begin{tabular}{ccc}
\hline Tested NiTi instruments & NCF (SD) & Fragment length (mm) (SD) \\
\hline ProFile $(n=40)$ & $365.25(75.64)^{c}$ & $2.5(0.4)$ \\
FlexMaster $(n=40)$ & $376.12(63.38)^{c}$ & $2.6(0.4)$ \\
Twisted File $(n=40)$ & $483.1(133.85)^{b}$ & $2.5(0.5)$ \\
Mtwo $(n=40)$ & $556.75(78.58)^{a}$ & $2.7(0.3)$ \\
\hline
\end{tabular}

$\mathrm{SD}=$ Standard deviation; * different letters in the same column indicate statistically significant difference

Table 2- Mean (SD) of the number of cycles to failure (NCF) and fragment lengths in $\mathrm{mm}(\mathrm{SD})$ of tested NiTi instruments according to the groups

\begin{tabular}{|c|c|c|c|}
\hline NiTi instruments & Groups & NCF (SD) & Fragment length $\mathrm{mm}(\mathrm{SD})$ \\
\hline \multirow[t]{4}{*}{ ProFile $(n=40)$} & Control & $396.5(77.46)$ & $2.6(0.4)$ \\
\hline & $5 \min \% 2.5 \mathrm{NaOCl}$ & $385.5(76.03)$ & $2.4(0.5)$ \\
\hline & $5 \min \% 2.5 \mathrm{NaOCl}+$ sterilization & $359(73.25)$ & $2.5(0.6)$ \\
\hline & $5 \mathrm{X}(5 \min \% 2.5 \mathrm{NaOCl}+$ sterilization $)$ & $320(61.55)$ & $2.6(0.2)$ \\
\hline \multirow[t]{4}{*}{ FlexMaster $(n=40)$} & Control & $379(70.54)$ & $3(0.4)$ \\
\hline & $5 \mathrm{~min} \% 2.5 \mathrm{NaOCl}$ & $378(53.39)$ & $2.5(0.4)$ \\
\hline & $5 \min \% 2.5 \mathrm{NaOCl}+$ sterilization & $374(70.82)$ & $2.7(0.3)$ \\
\hline & $5 X(5 \min \% 2.5 \mathrm{NaOCl}+$ sterilization $)$ & $373.5(67.37)$ & $2.5(0.4)$ \\
\hline \multirow[t]{4}{*}{ Twisted File $(n=40)$} & Control & $551.2(118.78)$ & $2.4(0.4)$ \\
\hline & $5 \min \% 2.5 \mathrm{NaOCl}$ & $526.5(105.8)$ & $2.4(0.2)$ \\
\hline & $5 \min \% 2.5 \mathrm{NaOCl}+$ sterilization & $439(148.16)$ & $2.6(0.4)$ \\
\hline & $5 \mathrm{X}(5 \min \% 2.5 \mathrm{NaOCl}+$ sterilization $)$ & $415.7(126.21)$ & $2.5(0.2)$ \\
\hline \multirow[t]{4}{*}{ Mtwo $(n=40)$} & Control & $599.5(79.46)$ & $2.8(0.2)$ \\
\hline & $5 \min \% 2.5 \mathrm{NaOCl}$ & $560(56.81)$ & $2.7(0.2)$ \\
\hline & $5 \min \% 2.5 \mathrm{NaOCl}+$ sterilization & $553(87.94)$ & $2.6(0.2)$ \\
\hline & $5 \mathrm{X}(5 \min \% 2.5 \mathrm{NaOCl}+$ sterilization $)$ & $514.5(73.76)$ & $2.6(0.2)$ \\
\hline
\end{tabular}

$\mathrm{SD}=$ Standard deviation 
FlexMaster, Twisted Files and Mtwo). The length of each fractured tip was also recorded.

\section{Statistical analysis}

Bartlett's test showed that the data did not provide preconditions of homogeneity of variance $(P<0.05)$ and were not normally distributed according to Anderson-Darling test $(P<0.05)$. Thus, the significances among the tested instruments were statistically analyzed with Kruskall-Wallis test $(P=0.05)$.

\section{RESULTS}

Kruskall-Wallis test indicated significant differences among the tested instruments in terms of NCF, P=0.000. The mean NCF of Mtwo (556.75) was higher than that of the Twisted File, Flexmaster and ProFile, 483.1, 376.12, 365.25, respectively (Table 1).

Kruskal-Wallis test showed that $\mathrm{NaOCl}$ immersion and autoclave sterilization have no effect on the NCF values of the tested instruments $(P>.05)$, when the effects of $\mathrm{NaOCl}$ immersion and autoclave sterilization on each instrument were searched (Table 2).

There were no statistically significant differences among the tested instruments in terms of the length of fractured segments, ( $P>.05)$ (Table 1). The mean lengths of the separated segments as measured from the instruments' tips were 2.7, 2.6, 2.5 and, $2.5 \mathrm{~mm}$ for Mtwo, FlexMaster, Twisted File and Profile, respectively (Table 1 ).

\section{DISCUSSION}

In the literature, there is still a debate regarding the impact of torsional stress and metal fatigue on the fracturing of $\mathrm{NiTi}$ rotary instruments. A number of studies have stated that fatigue is the predominant mechanism in material failure ${ }^{6,25}$. On the other hand, some authors have hypothesized that torsional failure occurs more frequently than flexural fatigue ${ }^{1,23}$.

The fatigue life of an instrument is determined by the radius and angle of curvature, which are associated with the diameter of the instrument in the region of maximum bending ${ }^{3}$. Thus, as the diameter of the instrument increases and the curvature radius of the canal decreases, the fatigue resistance of the rotary instruments decreases ${ }^{29}$. Other factors related to cyclic fatigue are the design and cross section of the instrument ${ }^{27,31}$, the working speed, torque and sterilization procedures ${ }^{4,5}$ and clinical usage ${ }^{3,8}$.

To simulate clinical conditions, the test protocol chosen for this study included dynamic immersion in $2.5 \% \mathrm{NaOCl}$, a maximum of five cycles of autoclave sterilization, and dynamic cyclic fatigue resistance testing. To remain within a realistic time-frame of clinical practice and considering the mean life of the control instruments (between $1 \mathrm{~min}, 51.36 \mathrm{~s}$ for Mtwo; and $1 \mathrm{~min}, 13.06 \mathrm{~s}$ for ProFile), $5 \mathrm{~min}$ was selected as the contact time of the solution with the instrument. The shaft of the instrument was not immersed in the irrigation solution, as is the case in root canal instrumentation procedures. This also served the purpose of avoiding galvanic corrosion phenomena ${ }^{4}$. A dynamic model that incorporates cyclic axial movement not only provides better simulation of the clinical environment but also lengthens the lifespan of the rotary files, because the compressive and tensile stresses are distributed along the instruments ${ }^{14,16}$. However, the NCF values obtained in the present study were lower than those obtained in other studies that used a dynamic model ${ }^{16,31}$. Those studies used an artificial canal that was not constructed based on the specific dimensions of each file group to ensure a suitable file trajectory. Therefore, the canal design they used may have affected the fatigue life and resulted in a wide variation in the fatigue life of the instruments tested, as was claimed by Plotino, et al. ${ }^{21}$ (2009).

The number of times that rotary instruments can be reused is suggested to vary between 1 and $10^{9,12}$, depending on the canal conditions and the type of instrument usage. Clinicians often recycle NiTi files owing to economic considerations ${ }^{11}$. In the present study, reuse for five times was simulated by five cycles of $\mathrm{NaOCl}$ immersion and autoclave sterilization.

In a recent study, Pedullà, et al. ${ }^{18}$ (2011) reported that static or dynamic immersion in $5 \% \mathrm{NaOCl}$ did not significantly reduce the cyclic fatigue resistance of Twisted Files, Mtwo, or Revo S SU. The authors attributed this to the location of the corrosion. If the corrosion is not in the maximum stress area, then, the instrument's resistance to cyclic fatigue probably cannot be reduced ${ }^{18}$. In another study, ProFile and $\mathrm{RaCe}$ instruments showed a reduction in fatigue resistance after immersion in a heated $\mathrm{NaOCl}$ solution for $1 \mathrm{~h}^{19}$.

In the present study, the results showed no significant difference for the instruments made of traditional NiTi alloy (ProFile, FlexMaster and Mtwo) before and after $\mathrm{NaOCl}$ immersion and autoclave sterilization. The findings of other studies that investigated autoclave sterilization effects on $\mathrm{NiTi}$ instruments were in agreement with the present results $^{11,20}$. On the other hand, Viana, et al. ${ }^{29}$ (2006) reported that the NCF of ProFile rotary instruments $(25,0.06)$ increased by $16 \%$ after five autoclave cycles in a temperature range $122-128^{\circ} \mathrm{C}$. Furthermore, it has been demonstrated that the five cycles of autoclave sterilization increased the microhardness of the NiTi endodontic instruments ${ }^{5}$. 
$\mathrm{NaOCl}$ immersion and autoclave sterilization also had no significant influence on the cyclic fatigue life of the heat-treated instruments (Twisted Files). In contrary, Hilfer, et al. ${ }^{11}$ (2011) found that repeated autoclaving significantly reduced the mean NCF of $25 / .06$ Twisted Files. As they explained it, $135^{\circ} \mathrm{C}$ is too low a temperature to produce a heat treatment effect, because it is known that such changes require a temperature of at least $170^{\circ} \mathrm{C}$, and that the maximum fatigue resistance can be obtained at $430-440^{\circ} \mathrm{C}^{15}$. Furthermore, multiple autoclave cycles have been reported to increase the depth of NiTi file surface irregularities, causing fatigue propagation $^{1,28}$. On the other hand, Plotino, et al. ${ }^{20}$ (2012) suggested that autoclave sterilization might improve the mechanical properties of instruments (K3 XF protypes, SybronEndo) that are manufactured using a type of heat treatment. The quality of manufacturing processes and different thermal treatments influence the performance of $\mathrm{NiTi}$ rotary instruments because NiTi is an alloy that is very sensitive to both the thermal and mechanical (machining) stresses that can occur during the manufacturing process ${ }^{20}$. A better arrangement of the crystal structure and changes in the percentage of phases of the alloy obtained via thermal treatment provide improvements in superelastic and plastic behaviors ${ }^{20}$. In the production process of Twisted Files, R-phase heat treatment technology modifies the crystalline structure, making it finer than that of traditionally processed materials, and it maximizes the flexibility and resistance to breakage without affecting the superelasticity ${ }^{16}$. Although Plotino, et al. ${ }^{20}$ (2012) reported that this new manufacturing technology enhanced the mechanical properties of instruments, the results of the present study did not confirm this finding. The traditional NiTi instrument, Mtwo, showed better cyclic fatigue resistance than the other traditional instruments, ProFile and FlexMaster, and the heattreated instrument, Twisted Files. Twisted Files showed better cyclic fatigue resistance than ProFile and FlexMaster, as reported previously ${ }^{16}$. Oh, et al. ${ }^{16}$ (2010) demonstrated that Twisted Files have fewer surface defects (microfractures) because of twisting, and a smaller cross-sectional area than ProFile instruments, characteristics that might result in their better fatigue resistance. However, scanning electron micrography (SEM) micrographs revealed wrinkle-like grooves because of twisting on the flute faces of Twisted Files, which may increase their level of stress during instrumentation ${ }^{31}$.

All tested instruments fractured at the point of the maximum flexure within the curved part of the artificial canal where the stress concentrates. As expected, the lengths of the fractured segments of the instruments were not influenced by the $\mathrm{NaOCl}$ immersion and autoclave sterilization.

\section{CONCLUSION}

The comparison within the conditions of this study showed that cyclic fatigue resistance of the tested $\mathrm{NiTi}$ instruments cannot be adversely affected by $\mathrm{NaOCl}$ immersion and autoclave sterilization. Production process (TwistedFiles) or design (Twisted Files, FlexMaster, Mtwo and ProFile) of the instruments can influence their cyclic fatigue resistance. However, further studies investigating the different factors which can affect the instruments cyclic fatigue resistance, fracture modes and different apparatus design with more similar features to root dentine are necessary.

\section{ACKNOWLEDGEMENTS}

We would like to thank Assistant Professor Dr. Özgür Koşkan for statistical analyses.

\section{REFERENCES}

1- Alapati SB, Brantley WA, Svec TA, Powers JM, Nusstein JM, Daehn GS. SEM observations of nickel-titanium rotary endodontic instruments that fractured during clinical Use. J Endod. 2005;31:40-3.

2- Arruda MP, Carvalho Junior JR, Miranda CE, Paschoalato C, Silva SR. Cleaning of flattened root canals with different irrigating solutions and nickel-titanium rotary instrumentation. Braz Dent J. 2009;20:284-9.

3- Bahia MG, Buono VT. Decrease in the fatigue resistance of nickel-titanium rotary instruments after clinical use in curved root canals. Oral Surg Oral Med Oral Pathol Oral Radiol Endod. 2005; 100:249-55.

4- Berutti E, Angelini E, Rigolone M, Migliaretti G, Pasqualini D. Influence of sodium hypochlorite on fracture properties and corrosion of ProTaper Rotary instruments. Int Endod J. 2006;39:693-9.

5- Chaves Craveiro de Melo M, Guiomar de Azevedo Bahia M, Lopes Buono VT. Fatigue resistance of engine-driven rotary nickeltitanium endodontic instruments. J Endod. 2002;28:765-9.

6- Cheung GS, Darvell BW. Fatigue testing of a NiTi rotary instrument. Part 2: Fractographic analysis. Int Endod J. 2007;40:619-25.

7- Gambarini G. Cyclic fatigue of nickel-titanium rotary instruments after clinical use with low- and high-torque endodontic motors. J Endod. 2001;27:772-4.

8- Gambarini G. Cyclic fatigue of ProFile rotary instruments after prolonged clinical use. Int Endod J. 2001;34:386-9.

9- Gambarini G, Grande NM, Plotino G, Somma F, Garala M, De Luca $M$, et al. Fatigue resistance of engine-driven rotary nickeltitanium instruments produced by new manufacturing methods. J Endod. 2008;34:1003-5.

10- Haikel Y, Serfaty R, Wilson P, Speisser JM, Allemann C. Mechanical properties of nickel-titanium endodontic instruments and the effect of sodium hypochlorite treatment. J Endod. 1998;24:731-5.

11- Hilfer PB, Bergeron BE, Mayerchak MJ, Roberts HW, Jeansonne BG. Multiple autoclave cycle effects on cyclic fatigue of nickeltitanium rotary files produced by new manufacturing methods. J Endod. 2011;37:72-4.

12- Inan U, Gonulol N. Deformation and fracture of Mtwo rotary nickel-titanium instruments after clinical use. J Endod. 2009;35:1396-9. 
13- Larsen CM, Watanabe I, Glickman GN, He J. Cyclic fatigue analysis of a new generation of nickel titanium rotary instruments. J Endod. 2009;35:401-3.

14- Li UM, Lee BS, Shih CT, Lan WH, Lin CP. Cyclic fatigue of endodontic nickel titanium rotary instruments: static and dynamic tests. J Endod. 2002;28:448-51.

15- Mize SB, Clement DJ, Pruett JP, Carnes DL Jr. Effect of sterilization on cyclic fatigue of rotary nickel-titanium endodontic instruments. J Endod. 1998;24:843-7.

16- Oh SR, Chang SW, Lee Y, Gu Y, Son WJ, Lee W, et al. A comparison of nickel-titanium rotary instruments manufactured using different methods and cross-sectional areas: ability to resist cyclic fatigue. Oral Surg Oral Med Oral Pathol Oral Radiol Endod. 2010;109:622-8.

17- O'Hoy PY, Messer HH, Palamara JE. The effect of cleaning procedures on fracture properties and corrosion of $\mathrm{NiTi}$ files. Int Endod J. 2003;36:724-32.

18- Pedullà E, Grande NM, Plotino G, Pappalardo A, Rapisarda E. Cyclic fatigue resistance of three different nickel-titanium instruments after immersion in sodium hypochlorite. J Endod. 2011;37:1139-42.

19- Peters OA, Roehlike JO, Baumann MA. Effect of immersion in sodium hypochlorite on torque and fatigue resistance of nickeltitanium instruments. J Endod. 2007;33:589-93.

20- Plotino G, Costanzo A, Grande NM, Petrovic R, Testarelli L, Gambarini G. Experimental evaluation on the influence of autoclave sterilization on the cyclic fatigue of new nickel-titanium rotary instruments. J Endod. 2012;38:222-5.

21- Plotino G, Grande NM, Cordaro M, Testarelli L, Gambarini G. A review of cyclic fatigue testing of nickel-titanium rotary instruments. J Endod. 2009;35:1469-76.

22- Sarkar NK, Redmond W, Schwaninger B, Goldberg AJ. The chloride corrosion behaviour of four orthodontic wires. J Oral Rehabil. 1983;10:121-8.
23- Sattapan B, Nervo GJ, Palamara JE, Messer HH. Defects in rotary nickel-titanium files after clinical use. J Endod. 2000;26:161-5.

24- Schäfer E. Effect of sterilization on the cutting efficiency of PVD-coated nickel-titanium endodontic instruments. Int Endod J. 2002;35:867-72.

25- Shen Y, Cheung GS, Peng B, Haapasalo M. Defects in nickeltitanium instruments after clinical use. Part 2: Fractographic analysis of fractured surface in a cohort study. J Endod. 2009;35:133-6.

26- Shen Y, Qian W, Abtin H, Gao Y, Haapasalo M. Effect of environment on fatigue failure of controlled memory wire nickeltitanium rotary instruments. J Endod. 2012;38:376-80.

27- Turpin YL, Chagneau F, Vulcain JM. Impact of two theoretical cross-sections on torsional and bending stresses of nickel-titanium root canal instrument models. J Endod. 2000;26:414-7.

28- Valois CR, Silva LP, Azevedo RB. Multiple autoclave cycles affect the surface of rotary nickel-titanium files: an atomic force microscopy study. J Endod. 2008;34:859-62.

29- Viana AC, Gonzalez BM, Buono VT, Bahia MG. Influence of sterilization on mechanical properties and fatigue resistance of nickel-titanium rotary endodontic instruments. Int Endod J. 2006;39:709-15.

30- Yahata Y, Yoneyama T, Hayashi Y, Ebihara A, Doi H, Hanawa T, Suda $\mathrm{H}$. Effect of heat treatment on transformation temperatures and bending properties of nickel-titanium endodontic instruments. Int Endod J. 2009;42:621-6.

31- Yao JH, Schwartz SA, Beeson TJ. Cyclic fatigue of three types of rotary nickel-titanium files in a dynamic model. J Endod. 2006;32:55-7. 